\title{
Maturation and Antigen Loading Protocols Influence Activity of Anticancer Dendritic Cells
}

\author{
Zh. K. Nazarkina ${ }^{a, b}, *$, A. Zajakina ${ }^{c}$, and P. P. Laktionov ${ }^{a, b}$ \\ ${ }^{a}$ Institute of Chemical Biology and Fundamental Medicine, Siberian Branch, Russian Academy of Sciences, \\ Novosibirsk, 630090 Russia \\ ${ }^{b}$ Meshalkin National Medical Research Center, Ministry of Health of the Russian Federation, Novosibirsk, 630055 Russia \\ ${ }^{c}$ Latvian Biomedical Research and Study Centre, Riga, LV1067 Latvia \\ *e-mail:zha_naz@niboch.nsc.ru \\ Received June 15, 2016; in final form, October 28, 2016
}

\begin{abstract}
The practical use of dendritic cell-based vaccines in anticancer therapy is limited by a lack of standards for dendritic cell (DC) generation, as well as standard procedures for controlling their activation and the technique of DC loading with nucleic acids encoding tumor antigens. Analyzing the currently available data, the most promising cocktails for DC maturation were selected and a comparative study of the cocktails and time of maturation on the capacity of DC to activate T-cell immune response has been performed. A study of the expression of surface markers and the production of IL-12, IL-6, and IL-10 cytokines, as well as the efficacy of T-cell activation showed that the use of the standard 7-day maturation protocol is preferable to the 4-day maturation protocol. Cocktails composed of TNF- $\alpha$, IL-1 $\beta$, IFN- $\alpha$, IFN- $\gamma$, and poly(I:C), as well as TNF- $\alpha$, IL-1 $\beta$, IFN- $\gamma$, R848, and PGE2 were shown to be the most efficient activators of DCs. A comparison of the efficacy of different methods of DNA transfection into DCs and RNA delivery using alphavirus vectors demonstrated the superiority of magnet-assisted transfection (MATra) to other protocols.
\end{abstract}

Keywords: dendritic cells, antigen delivery, cytotoxic T lymphocytes, anticancer immunotherapy

DOI: $10.1134 / \mathrm{S} 0026893317050132$

\section{INTRODUCTION}

Immunotherapy with dendritic cells is a relatively safe method that is much less toxic than standard chemotherapy and radiotherapy, but is not yet widely used in clinical practice, particularly due to the difficulty of obtaining DCs and the lack of a standard protocol for obtaining and quality control of DC-based preparations. The main advantage of DCs is that the antigenpresenting cells with specific characteristics required for the adequate development of the antitumor immune response can be obtained in vitro. Dendritic cells used for therapy are generally obtained from patient's peripheral blood monocytes. Immature DCs (iDCs) are loaded with tumor antigens in vitro followed by the induction of their maturation and subsequent administration into the body [1, 2]. Various combinations of cytokines and TLR (toll-like receptor) ligands are used to stimulate DC maturation. It should be noted that, in the majority of studies that

Abbreviations: DC, dendritic cells; CD, cluster of differentiation; IL, interleukin; TNF- $\alpha$, tumor necrosis factor $\alpha$; IFN- $\alpha$, interferon $\alpha$; PGE2, prostaglandin E2; GM-CSF, granulocyte-macrophage colony-stimulating factor; $\mathrm{MHC}$, major histocompatibility complex; SFV, Semliki forest virus; Sin, Sindbis virus, LPS, lipopolysaccharide. propose new techniques for DC maturation, the standard mixture of TNF- $\alpha$, IL-1 $\beta$, IL- 6 and PGE2 is used as a control $[1,3]$. However, this mixture cannot be considered optimal because it does not allow one to obtain immunogenic DCs producing IL-12p70. To induce DC maturation, Russian scientists most commonly use inexpensive, commercially available TNF- $\alpha$ $[4,5]$. Dendritic cells obtained in this case produce predominantly IL-12p40 and hardly produce IL-12p70, which is required to activate cytotoxic $\mathrm{T}$ lymphocytes [6]. It was found that the following cocktails are most promising to obtain mature immunogenic DCs: TNF- $\alpha$, IL-1 $\beta$, IFN- $\gamma$, R848, PGE2, and poly(I:C) [7]; LPS and IFN- $\gamma[8]$; and TNF- $\alpha$, IL- $1 \beta$, IFN- $\alpha$, IFN- $\gamma$, and poly(I:C) [9]. We compared the capacity of DCs obtaining by using these cocktails to activate T-cell immune response.

In addition to variations in the composition of cocktails, the incubation time of DCs in the presence of immunomodulators is also of principal importance for obtaining mature DCs $[10,11]$. We also evaluated the effect of time on the expression level of surface markers of DCs; on the production of IL-12, IL-6, and IL-10, as well as on the antigen-specific activity of $T$ cells stimulated by these DCs. 
The immune response can be activated using DCs loaded with tumor antigens, such as tumor-specific proteins and peptides, tumor cell lysates, apoptotic and necrotic tumor cells, as well as nucleic acids encoding tumor antigens [1]. Each of these approaches has advantages and disadvantages. The main drawback of the loading of DCs with tumor cells and their lysates is the presence of antigens that are also characteristic of normal tissues; the induction of the immune response against them may increase the nonspecific toxicity of vaccines or lead to an autoimmune process. The use of DNA- and RNA-containing constructs for DC loading has the following advantages: (1) a relatively simple construct design and the possibility of obtaining a sufficient amount of the immunogenic DNA/RNA; (2) the possibility of including the construct of genes that encode immunomodulatory molecules to increase the efficiency of the immune response induction; (3) processing nucleic acid-based constructs implies intracellular synthesis of polypeptides, as a result of which the antigens can be expressed, processed, and be presented in a complex with MHC molecules for a long time, inducing T-cell immune response; and (4) the possibility of creating polyepitopic constructs, which may comprise a large number of T-cytotoxic and T-helper epitopes, guiding peptides, and proteasomal degradation sites, which ensures the efficient processing and presentation of an antigen. The main problem in using nucleic acid-based constructs is their delivery into cells and efficient expression in DCs. With this in mind, we compared the effectiveness of the delivery of genetic material into DCs using metafectene, lipofectamine, magnetic particles MATra, and vectors based on alphaviruses, i.e., Semliki forest virus (SFV) and Sindbis virus (Sin).

The clinical efficacy of DC-based vaccines depends on many factors, such as the method of obtaining and the degree of maturity of DCs, the type of antigens used for DC loading, the stage of the disease, and the method of vaccine administration. The DC-based vaccine against prostate cancer, Sipuleucel-T, has been clinically tested and approved for use. This vaccine comprises autologous DCs derived from the peripheral blood of patients and loaded with a chimeric protein that consists of prostatic acid phosphatase and GM-CSF. The results of phase III of clinical trials of this vaccine showed that the average survival rate of patients increase by 4 months [12]. Currently, several DC-based vaccines are tested in phase III clinical trials. Unfortunately, data on these vaccines are limited. It is known that the Northwest Biotherapeutics company has developed the $\mathrm{DCVax}{ }^{\circledR}-\mathrm{L}$ vaccine against glioblastoma, which consists of immature DCs loaded with tumor cell lysate (https://www.clinicaltrials.gov/ct2/show/study/NCT00045968). Immature or half-mature DCs are often used to prepare antiglioblastoma vaccines [13]; however, mature DCs ensure a more efficient induction of immune and clinical response to vaccination [14].
The CLBS20 vaccine against melanoma, which includes iDCs loaded with irradiated tumor cells and GM-CSF $(500 \mu \mathrm{g})$ as an adjuvant, is also currently tested in phase-III clinical trials (https://www.clinicaltrials.gov/ct2/show/study/NCT01875653). The results of phase-I and -II clinical trials showed that this vaccine is well tolerated and increases the five-year survival of patients with metastatic melanoma [15]. In 2006, a phase III clinical trial was performed with an anti-melanoma vaccine comprising mature DCs, obtained by using the standard cocktail (TNF- $\alpha$, IL-1 $\beta$, IL-6 and PGE2), which were loaded with peptides restricted for MHC classes I and II. However, it was found that, in stage IV melanoma, this vaccine had no advantage over dacarbazine chemotherapy [16]. It is possible that the absence of a positive result is due to, inter alia, the use of an ineffective standard cocktail for DC maturation.

It should be noted that the clinical study of many DC-based vaccines began before the cocktails compared in our study were proposed. DC maturation and loading protocols will be required to obtain new generations of DC-based vaccines because the use of mature DCs capable of activating a cytotoxic immune response can significantly increase the effectiveness of therapy.

\section{MATERIALS AND METHODS}

Obtaining immature dendritic cells. Dendritic cells were prepared by differentiation from peripheral blood monocytes of healthy donors, the samples of which were provided by the Central Clinical Hospital, Siberian Branch, Russian Academy of Sciences. The study was performed in compliance with the principles of voluntariness and confidentiality in accordance with the Fundamentals of RF Legislation on health protection for citizens. Mononuclear cells were isolated from the peripheral blood by centrifugation in ficoll density gradient on LSM medium (MP Biomedicals, United States). Monocytes were isolated as a fraction of mononuclear cells that exhibit an increased adhesion to the surface of culture plastic. For this purpose, mononuclear cells $\left(50 \times 10^{6}\right.$ cells $)$ were cultured on a Petri dish with a diameter of $10 \mathrm{~cm}$ in $10 \mathrm{~mL}$ of RPMI-1640 medium (Sigma, United States) supplemented with $10 \%$ fetal bovine serum (FBS) (Gibco, United States) and $2 \mathrm{mM} L$-glutamine in a $\mathrm{CO}_{2}$ incubator at $37^{\circ} \mathrm{C}$ and $5 \% \mathrm{CO}_{2}$. After incubation for $1 \mathrm{~h}$, the nonadherent cells were frozen for subsequent isolation of T cells, and the Petri dish was gently rinsed twice with $1 \mathrm{~mL}$ of RPMI-1640. To obtain immature DCs that were then used to obtain fast 4d-DCs and standard 7d-DCs, monocytes were cultured in an RPMI-1640 medium supplemented with $10 \%$ FBS, $100 \mu \mathrm{g} / \mathrm{mL}$ penicillin, and $100 \mu \mathrm{g} / \mathrm{mL}$ streptomycin in the presence of recombinant GM-CSF (100 ng/mL) and human IL-4 $(25 \mathrm{ng} / \mathrm{mL})$ (Peprotech, United States) in a $\mathrm{CO}_{2}$ incu- 
bator for 2 and 5 days, respectively. On days 3 and 5 , fresh GM-CSF and IL-4 were added to the culture medium.

Preparation of MCF-7 cell lysate for DC loading. MCF-7 human breast adenocarcinoma cells (American Tissue Cell Collection, United States) were cultured in IMDM medium (Gibco) supplemented with $10 \% \mathrm{FBS}, 100 \mu \mathrm{g} / \mathrm{mL}$ penicillin, and $100 \mu \mathrm{g} / \mathrm{mL}$ streptomycin until $90 \%$ of the monolayer. After removal from Petri dishes, cell were washed with phosphate buffer and pelleted by centrifugation at $2000 \mathrm{rpm}$ for $8 \mathrm{~min}$. The pellet was resuspended in sterile water, the cells were lysed by freezing and thawing 5 times at -196 and $37^{\circ} \mathrm{C}$. The protein concentration was determined spectrophotometrically. The lysate was aliquoted and stored in at $-20^{\circ} \mathrm{C}$.

Obtaining mature dendritic cells. On days 3 and 6, iDCs were loaded with cell lysate. For this purpose, the cells were incubated for $2 \mathrm{~h}$ with MCF-7 lysate $(5 \mu \mathrm{g} / \mathrm{mL})$ in RPMI-1640 medium supplemented with $10 \%$ FBS. After incubation, the suspension was supplemented with GM-CSF, IL-4, and one of the four types of the cocktails used to induce the maturation of DCs-DC1, DC2, DC3, and DC4: (1) $40 \mathrm{ng} / \mathrm{mL}$ TNF- $\alpha$; (2) $200 \mathrm{ng} / \mathrm{mL}$ LPS and $50 \mathrm{ng} / \mathrm{mL}$ IFN- $\gamma$; (3) $10 \mathrm{ng} / \mathrm{mL}$ TNF- $\alpha, 10 \mathrm{ng} / \mathrm{mL}$ IL- $1 \beta, 5000 \mathrm{U} / \mathrm{mL}$ IFN- $\gamma, 1 \mu \mathrm{g} / \mathrm{mL}$ R848, and $250 \mathrm{ng} / \mathrm{mL}$ PGE2; and (4) $50 \mathrm{ng} / \mathrm{mL}$ TNF- $\alpha, 25 \mathrm{ng} / \mathrm{mL}$ IL-1 $\beta, 3000 \mathrm{U} / \mathrm{mL}$ IFN- $\alpha, 1000 \mathrm{U} / \mathrm{mL}$ IFN- $\gamma$, and $20 \mu \mathrm{g} / \mathrm{mL}$ poly(I:C). After the addition of cocktails, the cells were cultured for 2 days. The cocktails were prepared using reagents purchased from the following companies: TNF- $\alpha$, IFN- $\gamma$, and IL-1 were from Peprotech; PGE2, LPS (E. coli strain 0111:B4), and poly(I:C) were from Sigma; $\mathrm{R} 848$ was from Invitrogen, and IFN- $\alpha$ (Intron $\mathrm{A}^{\mathrm{R}}$ ) was from Schering-Plough (United States). The control samples of immature DCs, which were not loaded with the lysate, were cultured simultaneously with the experimental cells in the medium supplemented with GM-CSF and IL-4. The morphology of DCs was studied using an Axiovert 200 inverted optical microscope (Carl Zeiss, Germany) in the phase contrast mode at magnification $\times 200$ and $\times 400$.

Dendritic cell phenotyping. Forty-eight hours after the addition of the cocktail, DCs were centrifuged and stained with the antibodies to surface markers CD83, CD86, CD14 (Abcam, England), and HLA-DR (Sorbent, Russia). The number of stained cells was determined by flow cytometry with a Cytomics FC 500 flow cytometer (Beckman Coulter Inc.) using the software provided by the manufacturer.

Enzyme immunoassay. The content of IL-6, IL-10, IL-4, and IFN- $\gamma$ was determined by ELISA using the commercial reagent kits from Vector-Best (Russia), and the content of IL-12 was determined using the reagent kit from RD Systems (United States) in accordance with the protocol provided by the manufacturer. After maturation, DCs were seeded into wells of a 96-well plate at a density of $4 \times 10^{4}$ cells in $250 \mu \mathrm{L}$ and cultured in RPMI-1640 medium with $10 \%$ FBS for $24 \mathrm{~h}$. The cells were then centrifuged, and the concentration of IL-12, IL-6, and IL-10 in culture supernatants was determined. The concentration of IL-4 and IFN- $\gamma$ in the medium was determined 7 days after cocultivation of DCs and T cells.

T-cell stimulation by dendritic cells. After the adhesion of monocytes to plastic, the nonadherent cells of the mononuclear fraction of peripheral blood were frozen in the presence of $10 \%$ dimethyl sulfoxide at a cooling rate of $1^{\circ} \mathrm{C} / \mathrm{min}$ and stored at $-70^{\circ} \mathrm{C}$. One day before the addition of $\mathrm{T}$ cells to DCs, the nonadherent cells were thawed and cultured in RPMI-1640 medium with $10 \%$ FBS in a $\mathrm{CO}_{2}$ incubator at $37^{\circ} \mathrm{C}$ and $5 \% \mathrm{CO}_{2}$ for $24 \mathrm{~h}$. T cells were isolated by magnetic negative selection using the Easy Sep Human T-cell enrichment kit (Stem Cell, Canada) according to the manufacturer's recommendations. The purity of the cells was estimated by staining with antibodies to CD3+ (Sorbent, Russia) using flow cytometry. T cells $\left(1.5 \times 10^{6}\right)$ were added to DCs at a ratio of $5: 1$ and cultured in RPMI-1640 medium with $10 \%$ FBS in a $\mathrm{CO}_{2}$ incubator at $37^{\circ} \mathrm{C}$ and $5 \% \mathrm{CO}_{2}$ for 7 days. Then, the culture medium was collected to determine IL-4 and IFN- $\gamma$. T cells were restimulated with the same DCs and cultured for another 7 days. After 14 days of culturing, IFN- $\gamma$-ELISPOT-analysis (BD Biosciences, United States) was performed using MCF-7 cells as a source of antigens. The number of cells producing Granzyme B was assessed by flow cytometry using antibodies against granzyme B (BD Biosciences).

Assessment of T-cell proliferation. $T$ cells were incubated with $2.5 \mu \mathrm{M}$ fluorescent dye CFSE (Invitrogen) in phosphate buffer at $37^{\circ} \mathrm{C}$ for $15 \mathrm{~min}$. Then, the cells were centrifuged, resuspended in RPMI-1640 with $10 \% \mathrm{FBS}$, and incubated at $37^{\circ} \mathrm{C}$ for $30 \mathrm{~min}$. T cells $\left(3 \times 10^{5}\right)$ were added to DCs at a ratio of $5: 1$ and cultured in RPMI-1640 medium with $10 \%$ FBS in a $\mathrm{CO}_{2}$ incubator at $37^{\circ} \mathrm{C}$ and $5 \% \mathrm{CO}_{2}$ for 10 days. The fluorescence of cells was assessed by flow cytometry. The dividing cells were defined as cells for which fluorescence intensity was lower than in the control.

Plasmid DNA delivery into dendritic cells. Immature DCs were transferred to a 48 -well plate $\left(5 \times 10^{5}\right.$ cells per well). The cells were transfected with $1 \mu \mathrm{g}$ of the pmaxGFP plasmid (Amaxa, Germany) using lipofectamine $(2 \mu \mathrm{L}$ per well; Invitrogen $)$, metafectene (1 $\mu \mathrm{L}$ per well; Biontex, Germany), or MATra $(0.6 \mu \mathrm{L}$ of the reagent per well; PromoCell, Germany) according to the protocol recommended by the manufacturer. The optimal concentrations of transfection agents were selected earlier. After $4 \mathrm{~h}$ of incubation, the medium was replaced with a fresh one. The transfected DCs were cultured in a $5 \% \mathrm{CO}_{2}$ atmosphere at $37^{\circ} \mathrm{C}$ for $48 \mathrm{~h}$. 
Preparation of recombinant alphaviruses. BHK cells (American Tissue Cell Collection) were cultured in DMEM medium (Gibco) in the presence of $10 \%$ FBS in a $\mathrm{CO}_{2}$ incubator at $37^{\circ} \mathrm{C}$ and $5 \% \mathrm{CO}_{2}$. Replicationdeficient recombinant SFV and Sin expressing green fluorescent protein (GFP) were obtained by coelectroporation of $\mathrm{BHK}$ cells with a mixture of vector and helper RNA as described earlier [17]. The vector RNA (pSFV1/GFP and pSinrep5/GFP [18]) and helper RNA (pSFV/Helper and pSin/DH-EB $[19,20]$ ) were synthesized in vitro using respective plasmid linearized with the SpeI restriction enzyme as a template (Thermo Scientific, Lithuania). RNA transcription was performed in vitro using $3 \mu \mathrm{g}$ of linearized plasmid DNA and $40 \mathrm{U}$ of SP6-RNA polymerase (Thermo Scientific) in $50 \mu \mathrm{L}$ of the reaction mixture according to the manufacturer's protocol. RNA transcripts were capped during the transcription reaction by the addition of $1 \mathrm{mM}$ cap structure analogue $3^{\prime}-\mathrm{O}-\mathrm{Me}-\mathrm{m}^{7} \mathrm{G}\left(5^{\prime}\right) \mathrm{ppp}\left(5^{\prime}\right) \mathrm{G}$ (New England Biolabs, United Kingdom). The template DNA was degraded using RNase-free DNase (Thermo Scientific).

To pack GFP-producing RNA into the recombinant virus-like particles, vector RNA synthesized in vitro and helper RNA (pSFV1/GFP + pSFV/Helper and pSinrep5/GFP + pSin/DH-EB, respectively) were introduced into BHK cells ( $10^{7}$ cells) by coelectroporation (two pulses of $850 \mathrm{~V}, 25 \mu \mathrm{F}$ ) using a Bio-Rad Gene Pulser instrument (Bio-Rad, United States). After electroporation, cells were resuspended in DMEM medium with $10 \% \mathrm{FBS}$ and incubated at $33^{\circ} \mathrm{C}$ and $5 \% \mathrm{CO}_{2}$ for $48 \mathrm{~h}$. After incubation, the medium containing SFV and Sin was collected, centrifuged at $8000 \mathrm{~g}$, and frozen for later use. The titer of viruses was determined by infecting BHK cells with standard dilutions of virus and analyzing the GFP expression with a Leica DMIL fluorescence microscope (Leica Microsystems, Germany). The titer of SFV/GFP and SIN/GFP viruses was $2 \times 10^{7}$ infectious units $/ \mathrm{mL}$.

Infection of dendritic cells with viral particles. Cells were washed with RPMI-1640 or phosphate buffer DPBS with $\mathrm{Mg}^{2+}$ and $\mathrm{Ca}^{2+}$ ions (Gibco) and pelleted by centrifugation at $2000 \mathrm{rpm}$ for $8 \mathrm{~min}$. Cells were resuspended in RPMI-1640 or DPBS and transferred to sterile tubes $\left(2.5 \times 10^{5}\right.$ cells in $\left.100 \mu \mathrm{L}\right)$. Then, the cells were supplemented with $300 \mu \mathrm{L}$ of viral particles SFV/GFP or Sin/GFP at 10 - or 25 -fold dilution and incubated at $37^{\circ} \mathrm{C}$ for $1 \mathrm{~h}$. Then, the cells were pelleted by centrifugation, resuspended in fresh RPMI-1640 medium with $10 \% \mathrm{FBS}$, and incubated in a $5 \% \mathrm{CO}_{2}$ atmosphere at $37^{\circ} \mathrm{C}$ for $48 \mathrm{~h}$. Some cells were stained with PI to assess their viability. The proportion of the cells that express GFP and the proportion of cells labeled with PI was assessed by flow cytometry.

Statistical data processing. The results were processed using the Statistica 6.0 package (StatSoft, Inc., United States) including embedded Microsoft Excel functions. Results are represented as the arithmetic mean values calculated from three replicates and the standard error of the mean. Pairwise comparisons were performed for the following iDC groups: $\mathrm{iDC} / \mathrm{DC} 1$, iDC/DC2, iDC/DC3, and iDC/DC4. The statistical significance of differences was estimated by Student's $t$-test. Differences were considered significant at $p<0.05$.

\section{RESULTS}

\section{Search for Optimal Conditions for Obtaining Mature Dendritic Cells}

Dendritic cells were obtained by differentiation from peripheral blood monocytes of healthy donors with the HLA-A*0201 haplotype. The standard protocol for obtaining mature DCs includes culturing monocytes in the presence of GM-CSF and IL-4 for 5 days, the addition of a cocktail for maturation and the loading of immature DCs with antigens on day 6 followed by culturing the cells for $1-2$ days $[7,9,21]$. We compared the DCs obtained within 4 (4d-DC) and 7 (7d-DC) days. The most available TNF- $\alpha$ and the cocktails that are considered to be the most promising for obtaining mature immunogenic DCs were selected as inducers of DC maturation. Using these cocktails, namely, (1) TNF- $\alpha$ [6]; (2) LPS and IFN- $\gamma$ [8]; (3) TNF- $\alpha$, IL-1 $\beta$, IFN- $\gamma$, R848, and PGE2 [7]; and (4) TNF- $\alpha$, IL-1 $\beta$, IFN- $\alpha$, IFN- $\gamma$, and poly(I:C) [9], we derived mature DCs - 4d-DC1 and 7d-DC1, 4d-DC2 and 7d-DC2, 4d-DC3 and 7d-DC3, and 4d-DC4 and 7d-DC4, respectively. Thus obtained DCs had a characteristic polygonal shape with numerous branched processes.

The yield of DCs depended on the donor and was $(60-140) \times 10^{3}$ from $10^{6}$ mononuclear cells. No statistically significant differences in the DC yield when using the fast and standard maturation protocols have been found. The variations in the yield of DCs obtained using different protocols did not exceed 7\%, which was most likely due to the measurement error.

The degree of DC maturation was assessed by flow cytometry. In the course of maturation, DCs should lose the monocyte marker CD14, acquire the marker of mature DCs CD83, and express the costimulatory molecule CD86. The expression of CD83, CD86, CD14, and HLA-DR markers was evaluated 4 and 7 days after the initiation of culturing using monoclonal antibodies specific to these markers. The DC phenotyping data are shown in Fig. 1. The mean fluorescence intensity (MFI) values for DCs obtained using different protocols are summarized in Table 1. We found no significant differences in the expression profiles of surface markers of the fast and standard DCs obtained using the same cocktail. Culturing DCs in the presence of any of the cocktails led to the loss of the monocyte marker CD14 compared to the controls (iDCs). However, the level of expression of DC maturity markers CD83 and CD86 depended on the cock- 

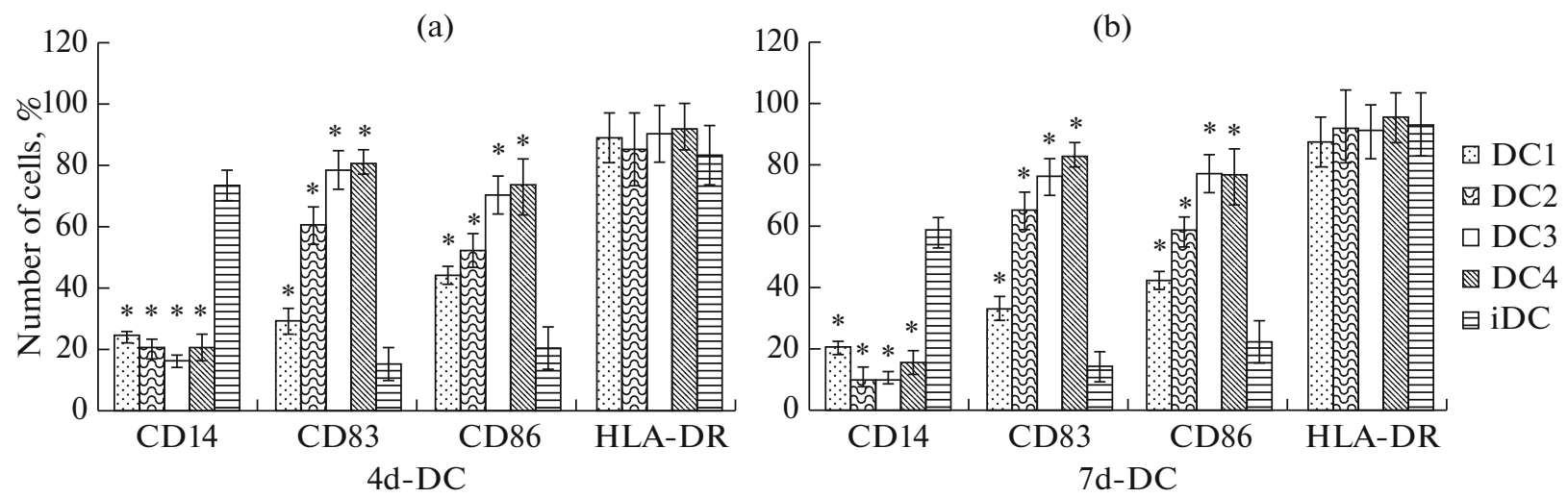

Fig. 1. Expression of receptors on DCs obtained using different protocols. Proportion of DCs that express CD14, CD83, CD86, and HLA-DR molecules on their surface was evaluated by flow cytometry after staining cells with antibodies. (a) DCs obtained for 4 days (4d-DC). (b) DCs obtained for 7 days (7d-DC). Results are represented as the mean values calculated from three independent experiments \pm standard error of the mean. Here and in Figs. $2-5,\left(^{*}\right)$ indicates that the values are statistically significant $(p<0.05)$ compared to the values in the group of immature DCs.

tail type. The minimum level was detected when using TNF- $\alpha$. The most effective maturation inducers were cocktails (3) and (4). The content (\%) of the CD83and CD86-positive cells in the case of using cocktail (2), which consists of IFN- $\gamma$ and LPS, was somewhat lower. Any differences in the expression of HLA-DR receptors in DC samples incubated with different cocktails were not detected.

We determined the levels of production of IL-12, IL- 6 , and IL- 10 by DCs. In the case of using cocktails (2), (3), and (4), the level of IL-12 production by the standard DCs obtained for 7 days was slightly higher compared to the fast DCs obtained for 4 days (Fig. 2a). The IL-12 concentration in the culture medium in the case of using these cocktails differed statistically significantly from the IL-12 concentration in the medium with iDCs. Differences in the IL-12 production by $\mathrm{DC} 1$ and iDC cells were nonsignificant. The level of IL-6 production by the fast DCs was more than twice lower compared to the standard DCs regardless of the maturation cocktail used (Fig. 2b). Level of IL- 6 production by the DC1 cells obtained using TNF- $\alpha$ did not exceed the level of IL- 6 production by iDCs. Maximum IL-6 level was ensured by cocktails (3) and (4). IL-10 production by DCs in the course of maturation increased with an increase in the time of obtaining mature DCs and was independed of the type of cocktail (Fig. 2c). The maximum ratio of IL-12 and IL-10 concentrations, which characterizes the proinflammatory potential of DCs, was observed when using cocktails (3) and (4) and, in the case of fast DCs, was almost three times higher compared to the standard DCs.

$\mathrm{T}$ cells were isolated from the fraction of peripheral blood mononuclear cells by using negative magnetic selection, which makes it possible to obtain a population that consists of more than $90 \% \mathrm{CD}^{+}$cells (according to flow cytometry data). The resulting $\mathrm{T}$ cells were cocultured with the mature DCs. To assess the degree of the activation of T cells by DCs of different types, we measured the content of IL-4 and IFN $\gamma$ in the culture medium and determined the number of

Table 1. Mean fluorescence intensity (MFI)* of dendritic cells obtained using different protocols

\begin{tabular}{|c|c|c|c|}
\hline \multirow{2}{*}{ Marker } & \multirow{2}{*}{ Cocktail type } & \multicolumn{2}{|c|}{ MFI, arb. units } \\
\hline & & $4 d-D C$ & $7 \mathrm{c}-\mathrm{DC}$ \\
\hline \multirow[t]{5}{*}{ CD14 } & DC1 & $148 \pm 18$ & $162 \pm 20$ \\
\hline & $\mathrm{DC} 2$ & $131 \pm 22$ & $96 \pm 14$ \\
\hline & DC3 & $116 \pm 17$ & $91 \pm 12$ \\
\hline & DC4 & $125 \pm 20$ & $150 \pm 16$ \\
\hline & iDC & $298 \pm 35$ & $221 \pm 24$ \\
\hline \multirow[t]{5}{*}{ CD83 } & DC1 & $157 \pm 14$ & $248 \pm 23$ \\
\hline & $\mathrm{DC} 2$ & $232 \pm 20$ & $342 \pm 27$ \\
\hline & DC3 & $626 \pm 48$ & $484 \pm 31$ \\
\hline & DC4 & $647 \pm 41$ & $629 \pm 45$ \\
\hline & iDC & $128 \pm 14$ & $148 \pm 16$ \\
\hline \multirow[t]{5}{*}{ CD86 } & DC1 & $180 \pm 17$ & $247 \pm 19$ \\
\hline & DC2 & $211 \pm 19$ & $361 \pm 27$ \\
\hline & DC3 & $348 \pm 27$ & $463 \pm 32$ \\
\hline & DC4 & $485 \pm 31$ & $512 \pm 36$ \\
\hline & iDC & $110 \pm 19$ & $154 \pm 25$ \\
\hline \multirow[t]{5}{*}{ HLA-DR } & DC1 & $498 \pm 28$ & $423 \pm 22$ \\
\hline & DC2 & $465 \pm 41$ & $520 \pm 41$ \\
\hline & $\mathrm{DC} 3$ & $490 \pm 36$ & $496 \pm 21$ \\
\hline & DC4 & $523 \pm 46$ & $547 \pm 33$ \\
\hline & iDC & $412 \pm 20$ & $462 \pm 40$ \\
\hline
\end{tabular}

* Data are represented as the mean values calculated from three independent experiments \pm standard error of the mean. 

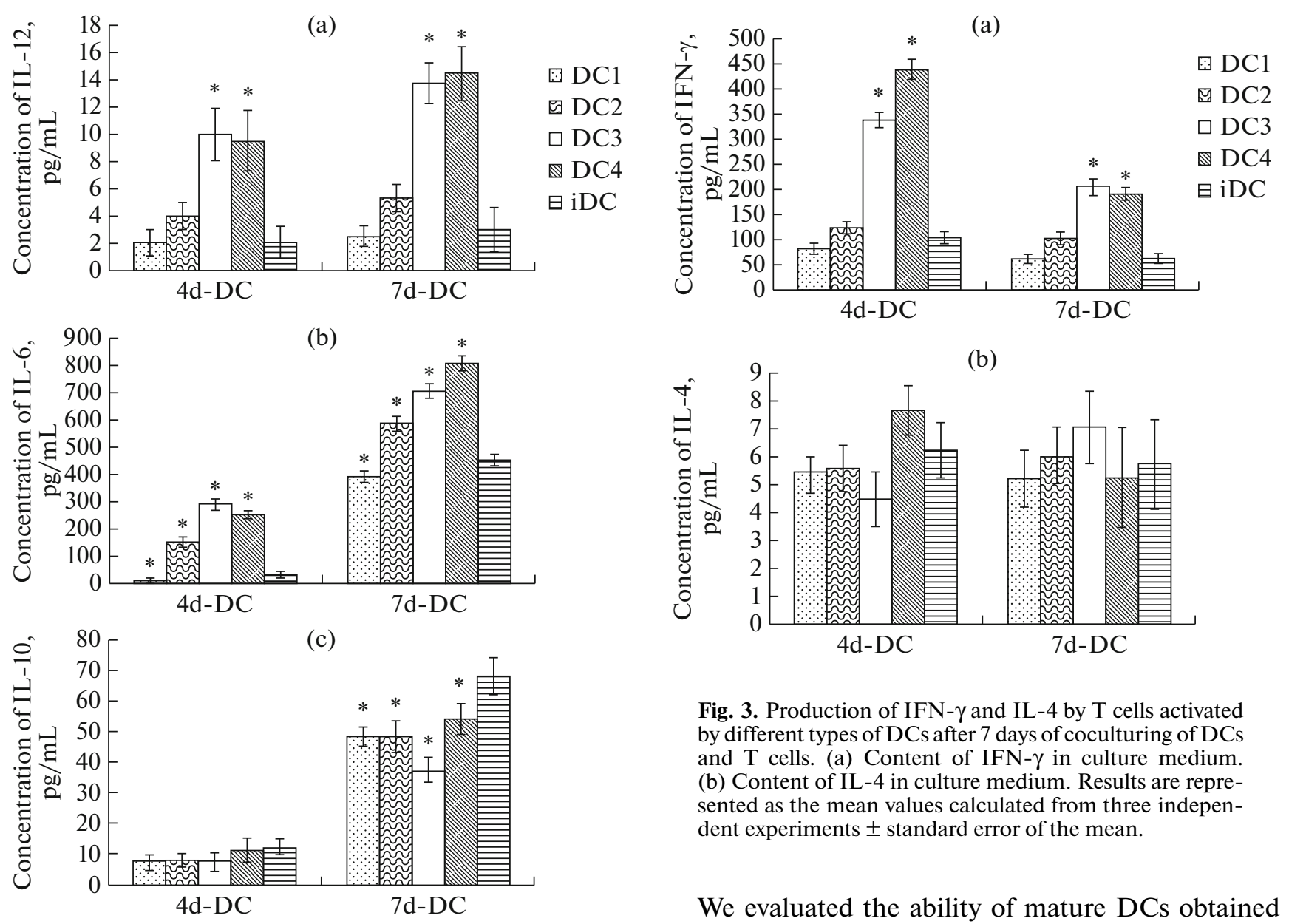

Fig. 3. Production of IFN- $\gamma$ and IL-4 by T cells activated by different types of DCs after 7 days of coculturing of DCs and $\mathrm{T}$ cells. (a) Content of IFN- $\gamma$ in culture medium. (b) Content of IL-4 in culture medium. Results are represented as the mean values calculated from three independent experiments \pm standard error of the mean.

Fig. 2. Production of interleukins by DCs obtained using different protocols. $(\mathrm{a}-\mathrm{c})$ Content of IL-12, IL-6, and IL10 in culture medium, respectively. Results are represented as mean values calculated from three independent experiments \pm standard error of the mean.

lymphocytes that synthesize IFN- $\gamma$ in response to the stimulation with MCF-7 cell lysates.

IFN- $\gamma$ is produced by the mature stimulated T cells in response to foreign antigens. The level of IFN- $\gamma$ secretion by the $T$ cells stimulated by the mature DCs obtained using cocktails (3) and (4) was significantly higher than in the case of cocktails (1) and (2) (Fig. 3a). When using cocktails (1) and (2), the secretion of IFN- $\gamma$ was similar to secretion in the case of the stimulation of T cells with the iDCs. The secretion of IFN- $\gamma$ by the $T$ cells stimulated with fast DCs exceeded its secretion by $\mathrm{T}$ cells stimulated with standard DCs.

Culturing $\mathrm{T}$ cells in the presence of mature DCs does not lead to an increase in the level of IL-4 (Fig. 3b), the main product of the T-helper type- 2 subpopulation. These data indicate that the obtained DCs promote Th1 (T helper type 1) polarization of T-cells, which favors the development of a cytotoxic immune response.

We evaluated the ability of mature DCs obtained using different cocktails to present antigens to T cells. $\mathrm{T}$ cells were cultured with DCs at a ratio of $5: 1$ for 14 days ( 7 days after the first stimulation of $T$ cells, they were restimulated with respective DCs). The antigen-specific activity of T cells against MCF-7 cells was assessed by the change in the number of $T$ cells that produce IFN- $\gamma$ in response to stimulation with MCF-7 cells by ELISPOT analysis. It was found that, in the case of using cocktails (3) and (4), an increase in the time of DC maturation from 4 to 7 days leads to an approximately 1.5 -fold increase in the number of IFN- $\gamma$-producing T cells (Fig. 4). In the case of cocktails (1) and (2), the number of IFN- $\gamma$-producing T cells only slightly differed from the control values. The number of IFN- $\gamma$-producing cells was maximum when $\mathrm{T}$ cells were stimulated by the DCs obtained using cocktail (4). When T cells were stimulated by the mature 7d-DC4 (-MCF-7) not loaded with the MCF-7 lysate, the number of IFN- $\gamma$-producing T cells did not statistically differ from the control values.

It was shown that the DCs obtained for 7 days using cocktail (4) stimulated the proliferation of T cells and the intracellular production of granzyme B by them. Due to the lack of donor material, the effect of other cocktails on these parameters was not assessed. Proliferation of $\mathrm{T}$ cells was determined using a method based on the inclusion of the intravital fluorescent dye 
CFSE, which binds to the intracellular proteins. In dividing cells, CFSE is distributed between the daughter cells, which leads to a decrease in the specific fluorescence. As can be seen in Fig. 5a, when T cells were stimulated with the mature DCs produced using cocktail (4), the proliferation of $\mathrm{T}$ cells was enhanced, which manifested in a decrease in their fluorescence intensity (Fig. 5a).

Granzyme B is a component of dense cytoplasmic granules secreted by activated cytotoxic $\mathrm{T}$ cells. According to the flow cytometry data, stimulation with mature 7d-DC4 leads to a twofold increase in the number of $T$ cells that produce granzyme $B$, whereas stimulation with immature DCs increases the proportion of these cells by only 1.5 times (Fig. 5b). These data show the effectiveness of using cocktail (4) to derive DCs capable of activating $T$ cells. Thus, it can be concluded that the cocktail consisting of TNF- $\alpha$, IL-1 $\beta$, IFN- $\alpha$, IFN- $\gamma$, and poly(I:C) is the most promising for creating DC-based vaccines.

\section{Loading of Dendritic Cells with Antigens Based on Plasmid DNA and Alphaviral Vectors}

The use of nucleic acids for loading DCs undoubtedly has a number of advantages. Indeed, tumor antigens can be delivered to DCs as the coding sequences in plasmid DNAs and viral RNA vectors, and their intracellular expression ensures the processing and presentation of antigens. To determine which method of nucleic acid delivery to cells ensures the maximum antigen expression, we compared the efficiency of the delivery of pmaxGFP plasmid DNA and viral particles encoding GFP. Dendritic cells were transfected with the plasmid DNA by three methods, i.e., by using lipofectamine LTX and metafectene as transfection reagents, as well as using the MATra magnet-assisted transfection kit. On day 6, iDCs were transfected with the pmaxGFP plasmid. After transfection, DC maturation was induced by addition of cocktail (4) to the medium. The proportion of GFP-expressing cells in the $7 \mathrm{~d}-\mathrm{DC} 4$ population was evaluated $48 \mathrm{~h}$ after transfection. Maximum level of transfection (up to $25 \%$ positive cells depending on the donor) was provided by the magnet-assisted transfection. The levels of transfection in the case of using lipofectamine and metafectene were 9 and $14 \%$, respectively. According to the cytotoxicity test, the treatment of cells with lipofectamine leads to the death of $30 \%$ of cells, whereas metafectene and MATra did not induce the death of DCs (Fig. 6b).

Alphaviral vectors can serve an alternative to plasmid DNAs for loading DCs with genetic material [22]. We infected DCs with SFV/GFP and SIN/GFP particles, then evaluated the proportion of GFP-expressing cells by flow cytometry. Cell viability was determined using the staining of cells with PI. The percentage of GFP-expressing DCs did not exceed 20\% (Fig. 6a). In addition, the virus particles proved to be extremely

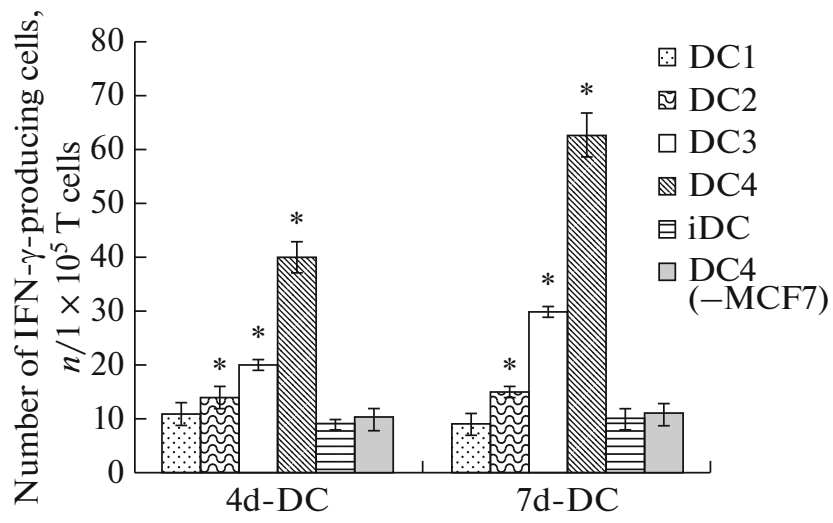

Fig. 4. Antigen-specific activity of T cells activated by different types of DCs with respect to MCF-7 cells. Number of IFN- $\gamma$-producing cells (per $1 \times 10^{5} \mathrm{~T}$ cells) in response to stimulation by MCF-7 cells were evaluated by ELISPOT analysis. Results are represented as the mean values calculated from three independent experiments \pm standard error of the mean.

toxic to DCs. Two days after transfection, 80-90\% DCs in which GFP expression was observed fluoresced in the PI channel; i.e., the integrity and viability of the functional membrane was lost (Fig. 6b). Thus, the most efficient method for plasmid DNA delivery to DCs among those tested in this study was the magnet-assisted transfection with the MATra kit.

\section{DISCUSSION}

Currently, there is no a commonly accepted protocol for assessing the effectiveness of activated DCs. The status and activity of DCs is determined by the expression level of surface markers, secreted immunoregulatory molecules, in a series of functional tests with certain advantages and disadvantages. In particular, the intensity of IL and IFN secretion only indirectly characterizes the ability to induce an immune response against specific antigens. Similarly, the receptor expression testifies to the differentiation status of DCs, but not their functional activity. Apparently, the function tests that directly show how effectively the DCs stimulate the activation of antigen-specific $\mathrm{T}$-cell immune response have the greatest informative value.

The maturity of DCs is evaluated not only by the level of surface markers, but also by ELISA, which makes it possible to determine the levels of cytokine secretion by DCs. Cytokine IL-12 is involved in the immune response, which contributes to the Th1 polarization of T cells [23]. In contrast, IL-10 inhibits the development of a cytotoxic immune response [1]. It was previously believed that IL-6 controls the development of the Th2 immune response and inhibits the Th1 response [24]. However, recent data are evidence that IL-6 does not affect the polarization of Th1 and Th2 but controls the proliferation and survival of these 
(a)
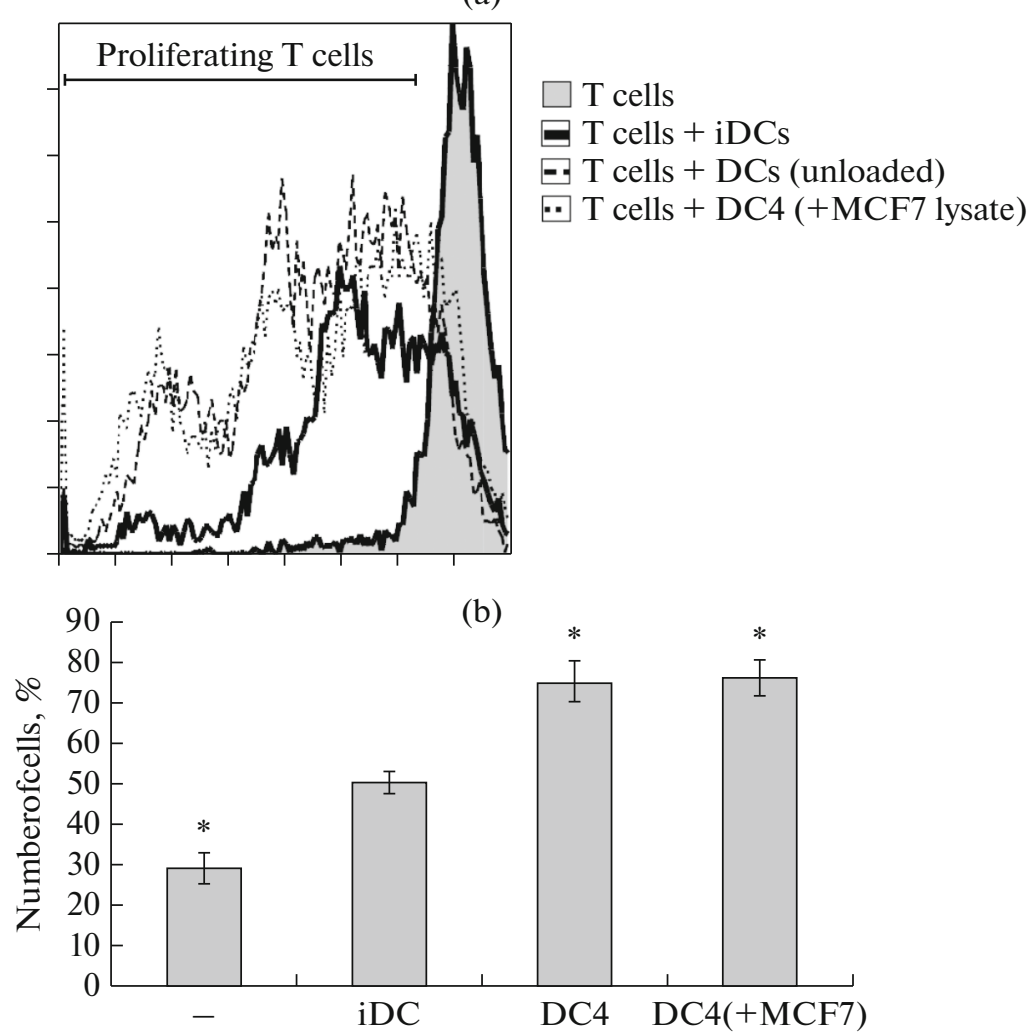

Fig. 5. Activation of T cells by 7d-DC4 cells. (a) Proliferation of T cells in the presence of DCs. CFSE-labeled T cells were cocultured with DCs for 10 days. Fluorescence intensity of the cell population was evaluated by flow cytometry. (b) Number of T cells producing granzyme B, determined by flow cytometry. Note. (-) indicates nonactivated T cells; iDCs are T cells activated by the immature DCs; DC4 are T cells activated by mature DCs not loaded with the lysate; DC4(+ MCF-7) are T cells activated by the mature DCs loaded with the MCF-7 lysate. Results are represented as the mean values calculated from three independent experiments \pm standard error of the mean.

cells [25]. In addition, IL-6 can suppress regulatory T cells [26]. Despite the high levels of surface marker expression by the fast DCs, the production of IL-12 and IL- 6 by the standard DCs was higher. However, the maximum ratio of IL-12/IL-10 concentrations in the case of using cocktails (3) and (4) was almost three times higher for the fast DCs compared to the standard DCs.

The secretion of IFN- $\gamma$ by the T cells stimulated with the fast DCs exceeded the secretion by the T cells stimulated with the standard DCs, which is in agreement with the published data. When the standard cocktail (TNF- $\alpha$, IL-1 $\beta$, IL-6, and PGE2) was used, the production of IFN- $\gamma$ by the T cells stimulated with the fast DCs was also higher compared to the T cells stimulated with the standard DCs [11]. It was shown that the standard DCs better activate the antigen-specific T cells. When cocktails (3) and (4) were used, the increase in the DC maturation time from 4 to 7 days entailed an approximately 1.5 -fold increase in the number of IFN- $\gamma$-producing T cells. It is possible that the number of IFN- $\gamma$-producing $T$ cells generated by the stimulation with the fast and standard DCs depends on the type of cocktail. The number of IFN- $\gamma$-producing
T cells in the case of using the cocktail consisting of TNF- $\alpha$, IL-1 $\beta$, IL-6, and PGE2 was higher in the case of their stimulation with the fast DCs than the standard cells [11]. According to our data, the use of the standard 7-day maturation protocol yields more active DCs. However, the fast 4-day protocol can be used at the preparatory stages of work because it allows one to obtain functionally active DCs with less material and time costs.

Based on the data obtained, we can conclude that the use of cocktails (3) (TNF- $\alpha$, IL-1 $\beta$, IFN- $\gamma$, R848, and PGE2) and (4) (TNF- $\alpha$, IL- $1 \beta$, IFN- $\alpha$, IFN- $\gamma$, and poly(I:C)) is most promising for creating DC-based vaccines. Cocktail (4) was proposed in 2004 [9]. Later, in 2007, Zobivalski et al. developed cocktail (3) and compared both cocktails; however, due to the lack of cellular material, the comparison was incomplete [7]. In particular, the effects of the two cocktails on the antigen-specific and cytotoxic activity of DC-stimulated $\mathrm{T}$ cells were not compared. We believe that the use of the combination of cocktail (4) and DC maturation for 7 days is optimum for obtaining immunogenic DCs. 
(a)

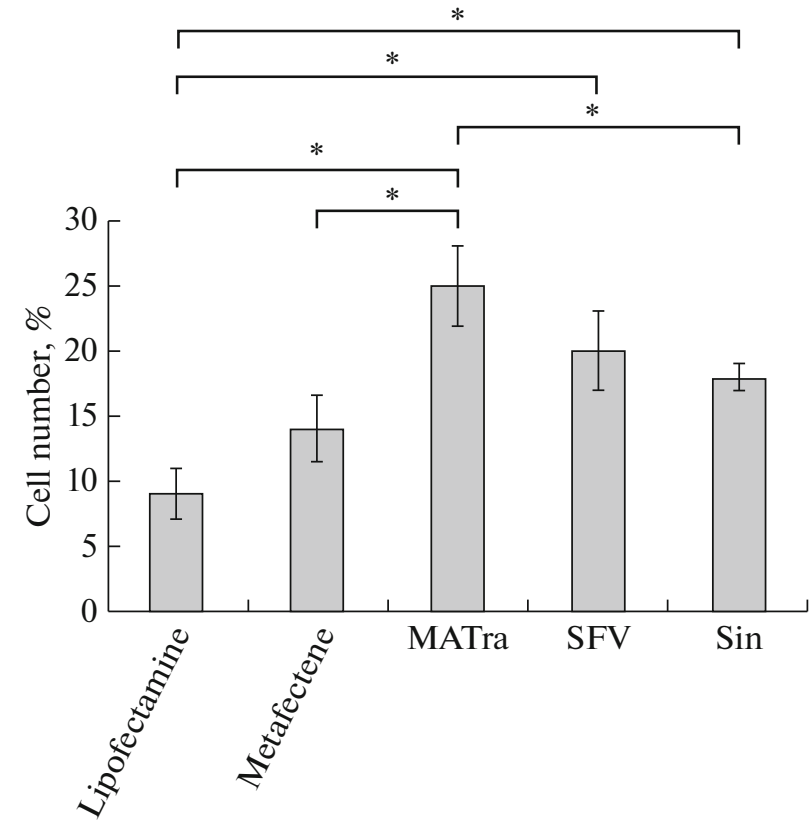

(b)

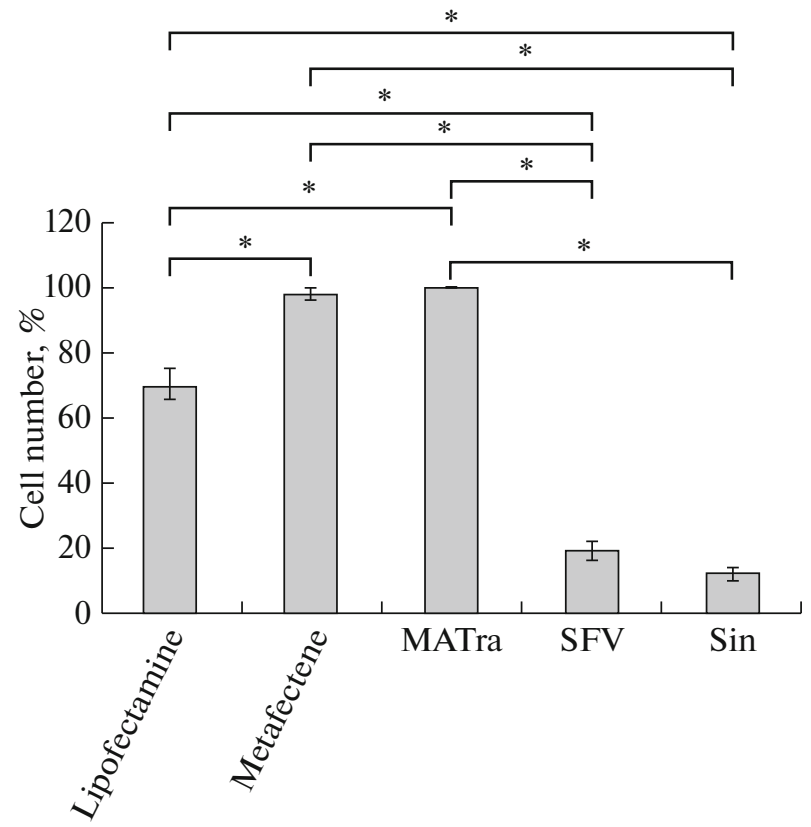

Fig. 6. Comparison of the efficiency of delivery of genetic material into DCs using metafectene, lipofectamine, and MATra magnetic particles, and vectors based on alphaviruses SFV and Sin. (a) Proportion of cells that express GFP protein. (b) Proportion of viable cells. Results are represented as mean values calculated from three independent experiments \pm standard error of the mean. $(*)$ Groups of significantly different values $(p<0.05)$.

It should be noted that the discrepancy between the results of different authors may be due to the use of drugs from different companies when preparing cocktails. Thus, the evaluation of the quality of a cocktail and its functional activity is a separate problem to be solved. The use of TNF- $\alpha$ as a single DC maturation inducer, despite its availability and low cost, is least advantageous. Our results confirm the earlier data [21]. Mature DCs prepared using TNF- $\alpha$ are characterized by low levels of expression of surface markers CD83 and CD86 and production of IL-12 and IL-6. In addition, more importantly, these DCs weakly activate effector $\mathrm{T}$ cells.

The problem of DNA/RNA delivery into DCs is very relevant. We studied the efficiency of different methods for delivery of genetic material into DCs using metafectene, lipofectamine, MATra magnetic particles, and alphaviral vectors. When using alphaviral vectors encoding the GFP protein, the majority of DCs die $48 \mathrm{~h}$ after infection, despite a relatively high level of GFP expression. Similar results are described in [27], where the penetration and expression of vectors based on the Venezuelan equine encephalomyelitis (VEE) virus in DCs was studied. It was found that, $48 \mathrm{~h}$ after the infection of DCs with the VEE/GFP viral particles, the proportion of live cells did not exceed $20 \%$, although in the first $24 \mathrm{~h}$ after infection approximately $70 \%$ DCs remained alive. Alphaviral vectors can probably be used in in vivo systems. Indeed, DC administered intradermally migrates to the lymph nodes within 12-24 h [28], and the interaction between DCs and T cells in vivo occurs within the first $24 \mathrm{~h}$ after immunization [29, 30]. Good results were obtained using alphaviral vectors in mice [22]. However, in some cases, the immunogenicity of antitumor vaccines cannot be studied in animal models. For example, the use of animal models is inappropriate when DCs are loaded with polyepitope DNAs/RNAs, which encode multiple epitopes of cancer antigens restricted for different HLA allomorphs because HLA molecules are unique to a particular taxon (family or even species). The immunogenicity of these vaccines is studied ex vivo; T cells are cultured in the presence of antigen-loaded DCs, after which their antigen-specific and cytotoxic activity is evaluated [4]. In this case, to stimulate $T$ cells, it is necessary that the loaded DCs remain viable for at least 1 week because, after loading DCs are cultured with T cells for 7-20 days (according to different data). The optimal method for loading DCs with plasmid DNA in ex vivo experiments is to use the MATra kit. This method allows to obtain viable DCs that most efficiently express the transfected antigen.

Thus, the standard 7-day maturation protocol yields more active DCs. Dendritic cells are activated most efficiency by cocktails that contain TNF- $\alpha$, IL- $1 \beta$, IFN- $\gamma$, R848, PGE2 and TNF- $\alpha$, IL- $1 \beta$, IFN- $\alpha$, IFN- $\gamma$, and poly(I:C). A comparison of the efficiency of different methods used to deliver nucleic acids that encode an antigen into DCs showed that the magnet- 
assisted transfection is the most suitable method for this purpose.

\section{ACKNOWLEDGMENTS}

This work was supported by the Basic Project of the Basic Research Program of State Academies of Science (VI.62.1.3, 0309-2016-0005), a project of the Russian Academy of Sciences (MRB no. 6.1), and the Biomedicine Latvian National Research Program.

\section{REFERENCES}

1. Skalova K., Mollova K., Michalek J. 2010. Human myeloid dendritic cells for cancer therapy: Does maturation matter? Vaccine. 28, 5153-5160.

2. Schuler G. 2010. Dendritic cells in cancer immunotherapy. Eur. J. Immunol. 40, 2123-2130.

3. Nazarkina Zh.K., Laktionov P.P. 2015. Preparation of dendritic cells for cancer immunotherapy. Biochemistry (Moscow). Suppl. Ser. B: Biomed. Chem. 8, 85-93.

4. Maksyutov A.Z., Lopatnikova Y.A., Kurilin V.V., et al. 2014. Efficiency studies of induced cytotoxic immune response of mononuclear cells by means of dendritic cells transfected by polyepitopic HER2/ErbB2 constructs. Med. Immunol. (Russia). 16, 417-424.

5. Nehaeva T.L., Baldueva I.A., Novik A.V., et al. 2014. Development and optimization of vaccines based on autologous dendritic cells (DC) is activated by cancertestis antigens for the treatment of patients with skin melanoma. J. Ural Med. Acad. Sci. 5, 92-98.

6. Morse M.A., Zhou L.J., Tedder T.F., et al. 1997. Generation of dendritic cells in vitro from peripheral blood mononuclear cells with granulocyte-macrophage-colony-stimulating factor, interleukin-4, and tumor necrosis factor-alpha for use in cancer immunotherapy. Ann. Surg. 226, 6-16.

7. Zobywalski A., Javorovic M., Frankenberger B., et al. 2007. Generation of clinical grade dendritic cells with capacity to produce biologically active IL-12p70. J. Transl. Med. 5, 18.

8. Dohnal A.M., Witt V., Hügel H., et al. 2007. Phase I study of tumor Ag-loaded IL-12 secreting semi-mature DC for the treatment of pediatric cancer. Cytotherapy, 9, 755-770.

9. Mailliard R.B., Wankowicz-Kalinska A., Cai Q., et al. 2004. alpha-type-1 polarized dendritic cells: a novel immunization tool with optimized CTL-inducing activity. Cancer Res. 64, 5934-5937.

10. Obermaier B., Dauer M., Herten J., et al. 2003. Development of a new protocol for 2-day generation of mature dendritic cells from human monocytes. Biol. Proceed. Online. 5, 197-203.

11. Bürdek M., Spranger S., Wilde S., et al. 2010. Three-day dendritic cells for vaccine development: Antigen uptake, processing and presentation. J. Transl. Med. 28 (8), 90.

12. Bol K.F., Schreibelt G., Gerritsen W.R., et al. 2016. Dendritic cell-based immunotherapy: State of the art and beyond. Clin. Cancer Res. 22, 1897-1906.

13. Wheeler C.J., Black K.L. 2009. DCVax-Brain and DC vaccines in the treatment of GBM. Expert Opin. Investig. Drugs. 18, 509-519.
14. Yamanaka R., Homma J., Yajima N. 2005. Clinical evaluation of dendritic cell vaccination for patients with recurrent glioma: results of a clinical phase I/II trial. Clin. Cancer Res. 11, 4160-4167.

15. Javed A., Sato S., Sato T. 2016. Autologous melanoma cell vaccine using monocyte-derived dendritic cells (NBS20/eltrapuldencel-T). Future Oncol. 12, 751-762.

16. Schadendorf D., Ugurel S., Schuler-Thurner B. 2006. Dacarbazine (DTIC. versus vaccination with autologous peptide-pulsed dendritic cells (DC) in first-line treatment of patients with metastatic melanoma: A randomized Phase III trial of the DC study group of the DeCOG. Ann. Oncol. 17, 563-570.

17. Vasilevska J., Skrastina D., Spunde K., et al. 2012. Semliki Forest virus biodistribution in tumor-free and 4T1 mammary tumor-bearing mice: A comparison of transgene delivery by recombinant virus particles and naked RNA replicon. Cancer Gene Therapy J. 19, 579-587.

18. Zajakina A., Vasilevska J., Zhulenkov D., et al. 2014. High efficiency of alphaviral gene transfer in combination with 5-fluorouracil in mouse mammary tumor model. BMC Cancer. 20(14), 460.

19. Liljestrom P., Garoff H. 1991. A new generation of animal cell expression vectors based on the Semliki Forest virus replicon. Biotechnology (NY). 9, 1356-1361.

20. Bredenbeek P.J., Frolov I., Rice C.M., et al. 1993. Sindbis virus expression vectors: packaging of RNA replicons by using defective helper RNAs. J. Virol. 67, 6439-6446.

21. Vopenkova K., Mollova K., Buresova I., et al. 2012. Complex evaluation of human monocyte-derived dendritic cells for cancer immunotherapy. J. Cell Mol. Med. 16, 2827-2837.

22. Lundstrom K. 2015. Alphaviruses in gene therapy. Viruses. 7, 2321-2333.

23. Colombo M.P., Trinchieri G. 2002. Interleukin-12 in anti-tumor immunity and immunotherapy. Cytokine Growth Factor Rev. 13, 155-168.

24. Rincón M., Anguita J., Nakamura T., et al. 1997. Interleukin(IL)-6 directs the differentiation of IL-4-producing CD4+ T cells. J. Exp. Med., 185, 461-469.

25. Hunter C.A., Jones S.A. 2015. IL-6 as a keystone cytokine in health and disease. Nat. Immunol. 16, 448-457.

26. Fujimoto M., Nakano M., Terabe F., et al. 2011. The influence of excessive IL-6 production in vivo on the development and function of Foxp3+ regulatory T cells. J. Immunol. 186, 32-40.

27. Moran T.P., Collier M., McKinnon K.P., et al. 2005. A novel viral system for generating antigen-specific $\mathrm{T}$ cells. J. Immunol. 175, 3431-3438.

28. Morse M.A., Coleman R.E., Akabani G., et al. 1999. Migration of human dendritic cells after injection in patients with metastatic malignancies. Cancer Res. 59, 56-58.

29. Mempel T.R., Henrickson S.E., von Andrian U.H. 2004. T-cell priming by dendritic cells in lymph nodes occurs in three distinct phases. Nature. 427, 154-159.

30. Hugues S., Fetler L., Bonifaz L., et al. 2004. Distinct T cell dynamics in lymph nodes during the induction of tolerance and immunity. Nat. Immunol. 5, 1235-1242.

Translated by M. Batrukova 